Psychological Topics, 30 (2021), 1, 115-123

Original Scientific Paper

UDC: $159.923 .2 .072(94)$

https://doi.org/10.31820/pt.30.1.6

613.81

\title{
What People Drink and Where They Drink It Can Inform Us About Their Personality
}

\author{
Peter K. Jonason ${ }^{1}$, Daniel Talbot ${ }^{2}$, and Joel Anderson ${ }^{3}$ \\ ${ }^{1}$ University of Padua, Padova, Italy \\ ${ }^{1}$ University of Cardinal Stefan Wyszynski, Warsaw, Poland \\ ${ }^{2}$ University of Notre Dame, Sydney, Australia \\ ${ }^{3}$ Australian Catholic University, Melbourne, Australia \\ ${ }^{3}$ La Trobe University, Melbourne, Australia
}

\begin{abstract}
Folk wisdom and research on personality inferences suggest one should be able to judge a person's personality based on their behaviour related to alcohol consumption. In a sample of Australians $(N$ $=1,232)$, we compared the utility of knowing where and what people prefer to consume alcohol to understand people's personality (broadly construed). Where people drank had limited utility; predicting hopelessness in those who drank at home more than at a licensed venue and the consumption of spirits for those high in extraversion at a licensed venue. In contrast, there were several differences in people's personality across drink preferences. For example, neuroticism was higher in cider and spirit drinkers than beer and wine drinkers. Results are framed within the personality inference literature and qualified by (1) the traditional beer-drinking culture of our sample and (2) the complex relationships between personality and any behaviour, including habits surrounding alcohol consumption.
\end{abstract}

Keywords: alcohol, Big Five, life satisfaction, resilience, hopelessness

\section{Introduction}

Inferences about people's personalities are quite hard at first acquaintance given the limited information available (Haselton \& Funder, 2006). Two behaviours that are overtly available in Western societies is what alcohol people drink and where

Peter K. Jonason, University of Padova, Department of General Psychology, Via Venezia, 12, 35131 Padova PD, Italy. E-mail: peterkarl.jonason@unipd.it

Authors' notes:

We thank Lion beer for funding the data from which this study was drawn; data that was previously reported in Jonason, Talbot, Cunningham, and Chonody (2020; Personality and Individual Differences). The first author was partially funded by the Polish National Agency for Academic Exchange (PPN/ULM/2019/1/00019/U/00001) and a grant from the National Science Centre of Poland (2019/35/B/HS6/00682). 
they drink it. Indeed, much of modern courtship involves the consumption of alcohol in licensed venues or at so-called house parties (Jonason, Foster et al., 2015), where people are likely to be making personality inferences to inform their mating decisions (Jonason, Garcia et al., 2015). Personality judgments are likely to have wide-reaching consequences and, thus, understanding what kinds of behaviours are informative about personality is an important task. In this brief report, we asked whether what people drink and where they drink it informs us about their personality.

Folk wisdom says that people who drink different kinds of alcohol have different personalities. ${ }^{1}$ Whether this has any truth to it warrants investigation. Personality traits predict real-world behaviours (Paunonen \& Ashton, 2001), including, but not limited to, the consumption of alcohol (Malouff et al., 2007). However, most research that considers the interaction between personality and alcohol tends to be confined to the Big Five traits (i.e., extraversion, conscientiousness, openness, neuroticism, and agreeableness), is concerned with alcohol consumption in general, and has failed to explore a range of alcoholic beverages (e.g., focusing solely on beer; Corcoran \& Segrist, 1998). Given that, there is little cause to make strong predictions, so relying on stereotypes about different alcohols might guide our thinking. For instance, beer - the most consumed alcoholic beverage around the world (Devaux \& Sassi, 2015) - might be consumed in social settings (Jonason, 2018). Therefore, beer may be associated with more psychological health (e.g., life satisfaction, resilience, and happiness in general) given the way social interaction improves psychological health (Leary, 1990). Wine, which is also likely to be consumed in social settings (Jonason, 2018), may, therefore, have similar benefits on people's psychological health (e.g., limited hopelessness and greater interpersonal trust). In addition, those who choose to consume wine tend to have greater cognitive abilities compared to those who prefer beer or spirits, irrespective of socioeconomic status (Martin, 2009; Mortensen et al., 2005). Given evidenced associations between cognitive abilities and personality traits such as openness and conscientiousness (Curtis et al., 2015), it is conceivable that wine drinkers may differ regarding these traits. In contrast, drinking spirits may reveal something more undesirable about a person. Indeed, spirits are often called "hard alcohol", which might suggest people consume it when in a state of stress; people high in neuroticism are in a relatively constant state of stress.

In addition, research has generally not considered how the location of consumption might be associated with many personality traits and might even interact with what they drink. For example, drinking alcohol at home may be a sign of social exclusion, which could be linked to hopelessness (Leary, 1990). Drinking at home has been linked to depression and has been treated as a destructive or problematic coping strategy (Grant et al., 2009; Ostafin \& Brooks, 2011). Depressed people may turn to alcohol when confronted with stress and negative affect (Martin,

\footnotetext{
${ }^{1}$ https://www.scoopwhoop.com/what-your-favorite-liquor-says-about-your-personality/
} 
2009). However, prior research was mostly concerned with clinical manifestations of depression, whereas we concern ourselves here with dispositional differences in a parallel of depression - hopelessness. Hopelessness is essentially the opposite of optimism (Beck et al., 1974). Nevertheless, we expect to find similar effects when considering hopelessness, that people who stay home to drink alcohol are more hopeless than those who drink at licensed venues (i.e., in public with others).

Can one draw inferences about people's personalities based on information about their drinking behaviours? Folk wisdom suggests this is so, but this needs empirical testing. In this study, we examine how people's personality traits (i.e., individual differences in happiness, resilience, hopelessness, interpersonal trust, and the Big Five personality traits) may differ as a function of their preferred alcohol and their preferred place to drink alcohol. We hope to add to the literature on personality inferences, but we suspect that, given the complicated relationships between personality and behaviours, any such links will be small in nature.

\section{Method}

\section{Participants and Procedure}

Participants were 1,232 Australian community members (50\% women) paid AUD $\$ 4$ through a market research firm. The average participant was 45.79 years old $(S D=16.71$; Range $=18-88) .{ }^{2}$ Of this sample, our study included those who drank alcohol $(1,045$ participants; $84 \%$ of the sample). Participant's reported drinking alcohol on average one day $(M=1.07 ; S D=1.44)$ per week. ${ }^{3}$ Participants were informed of the nature of the study, completed a series of self-report measures, and were thanked/debriefed upon completion. This study was approved by the ethics committee at Western Sydney University (H14099). Details on this project can be obtained at the OSF site: https://osf.io/dhp27/.

\section{Measures}

Alcohol consumption patterns were measured with two forced-choice questions. First, participants selected their preferred location for drinking (i.e., "at home" or "at a licensed venue"); most participants (> 77\%) of participants reported primarily drinking at home. Second, participants reported their preferred alcoholic drink (i.e., wine, beer, spirits, mixed drinks, cider, and none). Participants who reported "none" were excluded here; wine $(n=401)$ was preferred, followed by beer, $(n=375)$, spirits and mixed drinks $(n=193)$, and cider $(n=76)$.

\footnotetext{
${ }^{2}$ Age was correlated less than $|.25|$ with the other variables, thus it was excluded as a covariate.

${ }^{3}$ Consumption was correlated less than $|.15|$ with the other variables, thus it was excluded as a covariate.
} 
We measured individual differences in happiness with the Satisfaction with Life Scale (Diener et al., 1995). This scale is composed of five items where participants indicated their agreement $(1=$ strongly disagree; $5=$ strongly agree $)$ with items like "In most ways my life is close to my ideal". Items were summed to create an index of happiness (Cronbach's $\alpha=.89$ ).

We measured individual differences in resilience with the Brief Resilience Scale (Smith et al., 2008). The scale is composed of five items where participants indicated their agreement $(1=$ strongly disagree; $5=$ strongly agree $)$ with items like "I tend to bounce back quickly after hard times". Items were summed to create an index of resilience $(\alpha=.87)$.

We measured individual differences in hopelessness with the Hopelessness Scale (Beck et al., 1974). This scale is composed of five items where participants indicated their agreement $(1=$ strongly disagree; $5=$ strongly agree $)$ with items like "I might as well give up because I can't make things better for myself". Items were summed to create a measure of individual differences in symptoms of depression, hopelessness, and suicide risk $(\alpha=.94)$.

We measured interpersonal trust with the Rotter's Interpersonal Trust Scale (Robinson et al., 1991) by asking participants their agreement ( 1 = strongly disagree; 5 = strongly agree) with 25 questions (e.g., "Hypocrisy is on the increase in our society"). Items were summed to create an index of interpersonal trust $(\alpha=.81)$.

Lastly, we included a measure of the Big Five personality traits (Donnellan et al., 2006). Participants reported their agreement with four items per trait measuring extraversion $(\alpha=.80)$, neuroticism $(\alpha=.73)$, conscientiousness $(\alpha=.67)$, agreeableness $(\alpha=.79)$, and openness $(\alpha=.74)$. Items were summed to create indexes of each trait.

\section{Results}

We treated personality traits as dependent variables and used consumption patterns as independent variables in a natural groups design. A series of nine twoway ANOVAs examined the effects of drink-preference and drink-location on personality traits. We found only one interaction $\left(F[3,1042]=2.93, p<.05, \eta_{p}{ }^{2}<\right.$ $.01)$, suggesting that those who drink spirits at a licensed venue $(M=12.57, S D=$ 2.92) were more extraverted $(t[191]=2.18, p<.01$, Cohen's $d=0.69)$ than those who drink at home $(M=10.38, S D=3.42)$. However, for those who prefer beer, wine, or cider, location of consumption did not determine differences in extraversion levels. There were no other interactions $\left(F_{\mathrm{S}}<2.61\right)$. 


\section{Table 1}

Descriptive Statistics for Personality Traits Across Alcohol Preferences

\begin{tabular}{|c|c|c|c|c|}
\hline \multirow[b]{2}{*}{ Personality } & \multicolumn{4}{|c|}{ Mean $(S D)$} \\
\hline & $\begin{array}{c}\text { Beer } \\
(n=375)\end{array}$ & $\begin{array}{c}\text { Wine } \\
(n=401)\end{array}$ & $\begin{array}{c}\text { Cider } \\
(n=76)\end{array}$ & $\begin{array}{c}\text { Spirits/Mixed } \\
\text { Drinks } \\
(n=193)\end{array}$ \\
\hline Life satisfaction & $16.27(4.42)$ & $16.75(3.94)$ & $14.96(4.74)$ & $15.20(4.56)$ \\
\hline Resilience & $19.21 \quad(4.49)$ & $19.55(4.34)$ & $17.42 \quad(5.65)$ & $18.28(5.10)$ \\
\hline Hopelessness & $51.83(12.36)$ & $49.08(12.81)$ & $55.86(13.44)$ & $53.20(14.87)$ \\
\hline Interpersonal trust & $68.99(9.08)$ & $69.26(9.40)$ & $67.38(10.08)$ & 67.44 (9.67) \\
\hline Extraversion & 11.43 (3.31) & $11.30 \quad(3.24)$ & $10.70 \quad(3.28)$ & $10.90 \quad(3.43)$ \\
\hline Neuroticism & 11.14 & 10.79 (2.97) & $12.21 \quad(3.20)$ & $11.96(3.16)$ \\
\hline Conscientiousness & $14.17 \quad(2.73)$ & $14.63(2.76)$ & $13.91 \quad(3.01)$ & 13.87 (2.99) \\
\hline Openness & 13.78 (2.94) & $13.84(2.90)$ & $13.76 \quad(2.82)$ & $13.48 \quad(3.08)$ \\
\hline Agreeableness & $14.39 \quad(2.80)$ & $15.30 \quad(2.69)$ & $14.80 \quad(2.69)$ & $14.35 \quad(3.28)$ \\
\hline
\end{tabular}

Those who drink at home $(M=51.89, S D=13.64)$ were more hopeless $(F[1$, $\left.1044]=7.89, p<.01, \eta_{p}{ }^{2}<.01\right)$ than those who drink at a licensed venue $(M=49.66$, $S D=12.17)$. Those who primarily drink alcohol at a licensed venue $(M=11.73, S D$ $=3.36)$ were more extraverted $\left(F[1,1044]=8.53, p<.01, \eta_{p}{ }^{2}<.01\right)$ than those who drink at home $(M=11.10, S D=3.20)$. There were no other main effects for location $\left(F_{\mathrm{S}}<3.13\right)$.

Main effects for drink-type (i.e., four levels) were analyzed using Tukey's tests (see Table 1). Beer drinkers were more satisfied $\left(F[3,1042]=4.65, p<.01, \eta_{p}{ }^{2}<\right.$ $.02)$ than those who preferred spirits $(p<.05)$, and wine drinkers were more satisfied than cider $(p<.01)$ and spirits drinkers $(p<.01)$. Beer drinkers were more resilient $\left(F[3,1042]=5.49, p<.01, \eta_{p}{ }^{2}<.02\right)$ than cider drinkers $(p<.01)$, and wine drinkers were more resilient than cider $(p<.01)$ and spirits drinkers $(p<.05)$. Beer $(p<.05)$, cider $(p<.01)$, and spirits drinkers $(p<.01)$ were more hopeless $(F[3,1042]=4.18$, $\left.p<.01, \eta_{p}{ }^{2}<.02\right)$ than wine drinkers. Cider and spirits drinkers were more neurotic $\left(F[3,1042]=6.76, p<.01, \eta_{p}{ }^{2}=.02\right)$ than beer $(p s<.05)$ and wine drinkers $(p<$ $.01)$. Wine drinkers were more conscientious $\left(F[3,1042]=3.59, p<.05, \eta_{p}{ }^{2}=.01\right)$ than spirits drinkers $(p<.05)$. Wine drinkers were more agreeable $(F[3,1042]=5.62$, $\left.p<.01, \eta_{p}{ }^{2}<.02\right)$ than beer $(p<.01)$ and spirits $(p<.01)$ drinkers. There were no other differences by drink-type $(F \mathrm{~s}<3.19)$.

\section{Discussion}

One cannot observe people's personality, but instead, one can observe some behavioural residuals of personality and use these behaviours to draw inferences with some degree of error - about a person's traits (Haselton \& Funder, 2006). Generally, we found that the location of alcohol consumption was a limited indicator 
of personality, except in the case of hopelessness, and that those who primarily drink spirits at a licenced venue (compared to at home) tend to be more extraverted. Drinking at home may be destructive or problematic coping strategy (Grant et al., 2009; Ostafin \& Brooks, 2011). Depressed people, who likely experience higher levels of hopelessness, may turn to alcohol as a way of managing negative affective states because they lack the social support they need to have healthier ways of coping or they may merely be isolating as a function of their depression and drinking as they would otherwise. The effect for extraversion suggests that extraverted people may view spirits as something to drink with friends in the form of, for instance, doing "shots", but not at home (ostensibly alone).

In contrast, drink-choice proved to be a better indicator of personality. We found that beer and wine drinkers were the most satisfied with life and the most resilient, whereas wine drinkers were the most agreeable and conscientious, and the least hopeless, whilst cider and spirit drinkers were the most neurotic. Wine appears especially likely to be linked with social and personally desirable traits, whereas spirits drinking may be committed by those who are neurotic and linked to extraversion when consumed at a licensed venue compare to at home. Beer - the "drink of the people" - may be linked to greater satisfaction with life and resilience because beer drinking is a form of relaxation or leisurely activity (e.g., at a Sunday barbecue), whereas drinking spirits may be a more serious manifestation of drinking often done under duress (e.g., a shot of whiskey to calm the nerves). Drinking beer and wine, as opposed to other types of alcohol, may be linked with social activities which may create the life satisfaction/resilience effects we detected.

\section{Limitations and Conclusions}

This study provides a reasonably comprehensive answer to whether one can make inferences about someone's personality based on their drinking behaviours. The sample is limited in several ways. First, because it was a W.E.I.R.D. (Henrich et al., 2010) sample, it is unclear how well these effects will generalize. Second, the sample is limited specifically to an Australian context which has traditionally been a "beer culture" (Pettigrew, 2002) although there may have been a shift away from this in recent years. ${ }^{4}$ Third, our study did not consider the interaction of different personality types and drink choice. Stronger associations between drink choice and personality might have been found if interactions between personality traits were examined, for instance, the interaction between neuroticism and conscientiousness. This would require a different statistical approach, like logistic regression. Another limitation might be that we failed (by restrictions imposed from the data source) to consider traits like the so-called Dark Triad (i.e., psychopathy, narcissism, and

4 https://www.aihw.gov.au/reports/phe/221/alcohol-tobacco-other-drugs-australia/contents/ drug-types/alcohol 
Machiavellianism) which may be particularly toxic in interpersonal and organizational contexts, may be related to the consumption of alcohol, and, therefore, may be important inference one wants to make from consumption patterns (Koehn et al., 2019). Alternatively, we failed to examine the deleterious effects of alcohol consumption in people's lives, but this is already a well-researched topic (Haynes et al., 2005). Our data was cross-sectional, limiting our interpretations to how personality was correlated with preferences only. And last, Australia is a multicultural nation with, for instance, various religious denominations, but we were restricted, by the data source, from enquiring about religious affiliation. However, if religious denominations have an influence on venue or beverage choice, a mechanism to link them and personality traits seems like viable option.

We attempted to better understand the relationship between personality traits and where and what people drink. We focused on mean-level differences in personality as a function of self-reported alcohol-related behaviours. In general, our results revealed limited inferential utility of knowing where someone drinks, but what they drink may be relatively more useful. However, as a rule, beverage and location choice may be limited - on their own - to understand people's personality despite folk wisdom. Instead, such choices may simply reveal a person's drinking patterns. Alcohol-related choices may be too narrow to get a good picture of people's personality on their own, but coupled with other behaviours, they collectively may allow for more reliable inferences of personality.

\section{References}

Beck, A. T., Weissman, A., Lester, D., \& Trexler, L. (1974). The measurement of pessimism: The Hopelessness Scale. Journal of Consulting and Clinical Psychology, 42, 861-865. https://doi.org/10.1037/h0037562

Corcoran, K. J., \& Segrist, D. J. (1998). Fear of negative evaluation and gender interact to predict alcoholic beverage preference. Addictive Behaviors, 23, 509-515. https://doi.org/10.1016/S0306-4603(98)00021-5

Curtis, R. G., Windsor, T. D., \& Soubelet, A. (2015). The relationship between Big-5 personality traits and cognitive ability in older adults - A review. Aging, Neuropsychology, and Cognition, 22, 42-71. https://doi.org/10.1080/13825585.2014. 888392

Devaux, M., \& Sassi, F. (2015). Trends in alcohol consumption in OECD countries. In F. Sassi (Ed.), Tackling harmful alcohol use: Economics and health policy (pp. 39-59). OECD Publishing. https://doi.org/10.1787/9789264181069

Diener, E., Emmons, R. A., Larsen, R. Y., \& Griffin, S. (1995). The Satisfaction with Life Scale. Journal of Personality Assessment, 49, 71-75. https://doi.org/10.1207/ s15327752jpa4901_13 
Donnellan, M. B., Oswald, F. L., Baird, B. M., \& Lucas, R. E. (2006). The Mini-IPIP scales: Tiny-yet-effective measures of the Big Five factors of personality. Psychological Assessment, 2, 192-203. https://doi.org/10.1037/1040-3590.18.2.192

Grant, V. V., Stewart, S. H., \& Mohr, C. D. (2009). Coping-anxiety and coping-depression motives predict different daily mood-drinking relationships. Psychology of Addictive Behaviors, 23, 226-237. https://doi.org/10.1037/a0015006

Haselton, M. G., \& Funder, D. C. (2006). The evolution of accuracy and bias in social judgment. In M. Schaller, J. A. Simpson, \& D. T. Kenrick (Eds.) Evolution and Social Psychology (pp. 15-37). Psychosocial Press.

Haynes, J. C., Farrell, M., Singleton, N., Meltzer, H., Araya, R., Lewis, G., \& Wiles, N. J. (2005). Alcohol consumption as a risk factor for anxiety and depression: Results the longitudinal follow-up of the Nation Psychiatric Morbidity Survey. The British Journal of Psychiatry, 187, 544-551. https://doi.org/10.1192/bjp.187.6.544

Henrich, J., Heine, S. J., \& Norenzayan, A. (2010). The weirdest people in the world? Behavioral and Brain Sciences, 33, 61-83. https://doi.org/10.1017/ S0140525X0999152X

Jonason, P. K. (2018). Where everyone knows your name: The social and psychological value of having a "local" in Australia. White paper prepared for Lion Beer. Available from http://nebula.wsimg.com/baf3b01ac5276109b3afcdf9b44f6eb9?AccessKeyId=551B83 7F51991F7BBBCC\&disposition=0\&alloworigin=1

Jonason, P. K., Foster, J. D., McCain, J., \& Campbell, W. K. (2015). Where birds flock to get together: The who, what, where, and why of mate searching. Personality and Individual Differences, 80, 76-84. https://doi.org/10.1016/j.paid.2015.02.018

Jonason, P. K., Garcia, J., Webster, G. D., Li, N. P., \& Fisher, H. (2015). Relationship dealbreakers: What individuals do not want in a mate. Personality and Social Psychological Bulletin, 41, 1697-1711. https://doi.org/0.1177/0146167215609064

Koehn, M. A., Okan, C., \& Jonason, P. K. (2019). A primer on the Dark Triad traits. Australian Journal of Psychology, 71, 7-15. https://doi.org/10.1111/ajpy.12198

Leary, M. R. (1990). Responses to social exclusion: Social anxiety, jealousy, loneliness, depression, and low self-esteem. Journal of Social and Clinical Psychology, 9, 221229. https://doi.org/10.1521/jscp.1990.9.2.221

Malouff, J. M., Thorsteinsson, E. B., Rooke, S. R., \& Schutte, N. S. (2007). Alcohol involvement and the five-factor model of personality: A meta-analysis. Journal of Drug Education, 37, 277-294. https://doi.org/10.2190/DE.37.3.d

Martin, C. R. (2009). Personality characteristics associated with drinking and beverage preference. In V. R. Preedy (Ed.), Beer in health and disease prevention (pp. 171-176). Academic Press. https://doi.org/10.1016/B978-0-12-373891-2.X0001-6

Mortensen, L. H., Sørensen, T. I., \& Grønbæk, M. (2005). Intelligence in relation to later beverage preference and alcohol intake. Addiction, 100(10), 1445-1452. 
Ostafin, B. D., \& Brooks, J. J. (2011). Drinking for relief: Negative affect increases automatic alcohol motivation in coping-motivated drinkers. Motivation and Emotion, 35, 285 295. https://doi.org/10.1007/s11031-010-9194-5

Paunonen, S. V., \& Ashton, M. C. (2001). Big Five factors and facets and the prediction of behavior. Journal of Personality and Social Psychology, 81, 524-539. https://doi.org/ 10.1037/0022-3514.81.3.524

Pettigrew, S. (2002). A grounded theory of beer consumption in Australia. Qualitative Market Research, 5, 112-122. https://doi.org/10.1108/13522750210423814

Robinson, J. P., Shaver, P. R., \& Wrightsman, L. S. (1991). Measures of personality and social psychological attitudes. Academic Press.

Smith, B. W., Dalen, J., Wiggins, K., Tooley, E., Christopher, R., \& Bernard, J. (2008). The brief resilience scale: Assessing the ability to bounce back. International Journal of Behavioral Medicine, 15, 194-200. https://doi.org/10.1080/10705500802222972

Received: June 3, 2020 
